DOI: $10.15393 /$ j3.art.2018.4350

UDC 519.71, 517.977, 517.44

A. A. Hamoud, K. P. Ghadle

\title{
THE APPROXIMATE SOLUTIONS OF FRACTIONAL VOLTERRA - FREDHOLM INTEGRO-DIFFERENTIAL EQUATIONS BY USING ANALYTICAL TECHNIQUES
}

\begin{abstract}
This paper demonstrates a study on some significant latest innovations in the approximation techniques to find the approximate solutions of Caputo fractional Volterra-Fredholm integro-differential equations. To apply this, the study uses Adomian decomposition method and modified Laplace Adomian decomposition method. A wider applicability of these techniques is based on their reliability and reduction in the size of the computational work. This study provides analytical approximate to determine the behavior of the solution. It proves the existence and uniqueness results and convergence of the solution. In addition, it brings an example to examine the validity and applicability of the proposed techniques.
\end{abstract}

Key words: Adomian decomposition method, Laplace transform, Volterra-Fredholm integro-differential equation, Caputo fractional derivative

2010 Mathematical Subject Classification: 26A33, 49M27

1. Introduction. In this paper, we consider the Caputo fractional Volterra-Fredholm integro-differential equations of the form:

${ }^{c} D^{\alpha} y(x)=g(x)+a(x) y(x)+\int_{0}^{x} K_{1}(x, t) F_{1}(y(t)) d t+\int_{0}^{1} K_{2}(x, t) F_{2}(y(t)) d t$,

with the initial conditions

$$
y^{(i)}(0)=\delta_{i}, \quad i=0,1, \cdots, n-1,
$$

(C) Petrozavodsk State University, 2018

$$
\text { (cc) BY-NC }
$$


where $n-1<\alpha \leq n, n \in \mathbb{N}, y:[0,1] \longrightarrow \mathbb{R}$ is the continuous function to be determined, $g, a:[0,1] \longrightarrow \mathbb{R}$ and $K_{i}:[0,1] \times[0,1] \longrightarrow \mathbb{R}, i=1,2$, are continuous functions. $F_{i}: \mathbb{R} \longrightarrow \mathbb{R}, i=1,2$, are Lipschitz continuous functions. The fractional derivative ${ }^{c} D^{\alpha}$ is understood here in the Caputo sense.

The fractional integro-differential equations have attracted much more interest of mathematicians and physicists because they provide an efficiency for the description of many practical dynamical problems arising in engineering and scientific disciplines such as, physics, biology, electrochemistry, chemistry, economy, electromagnetic, control theory and viscoelasticity [3, 19, 20]. In recent years, numerous papers have been concentrating on the development of numerical and analytical techniques for fractional integro-differential equations. There are many approaches for seeking exact solutions of linear and nonlinear equations, for example, Al-Samadi and Gumah [3] applied the homotopy analysis method for fractional SEIR epidemic model, Jafarian et al. 14 applied successfully artificial neural networks approach to solve fractional order Volterra integrodifferential equations. Also, some comparisons had been made between this iterative approach and another traditional technique. The obtained results reveal that this method is very effective, Momani [19] and Qaralleh 20] applied Adomian polynomials to solve fractional integro-differential equations and systems of fractional integro-differential equations, Kadem and Kilicman [15] utilized the HPM and VIM methods for integrodifferential equation of fractional order with initial-boundary conditions, Yang [21] used the hybrid of block pulse function and Chebyshev polynomials to solve nonlinear Fredholm fractional integro-differential equations, Yang and Hou 22 applied the Laplace decomposition method to solve the fractional integro-differential equations, Mittal and Nigam [18] utilized the Adomian decomposition method to approximate solutions of fractional integro-differential equations, and Ma and Huang [17] applied hybrid collocation method to study integro-differential equations of fractional order. Moreover, properties of the fractional integro-differential equations have been studied by several authors [4, 12, 16].

The main objective of the present paper is to study the behavior of the solution that can be formally determined by such analytical approximation methods as the Adomian decomposition method and the modified Laplace Adomian decomposition method. Moreover, we prove the convergence and uniqueness solution of the fractional Volterra-Fredholm integro-differential equation. 
The rest of the paper is organized as follows. In section 2 some preliminaries and basic definitions related to fractional calculus and Laplace transform are recalled. In section 3 Adomian decomposition method is constructed for solving fractional Volterra-Fredholm integro-differential equations. In section 4 modified Laplace Adomian decomposition method is constructed for solving Volterra-Fredholm integro-differential equations of fractional order. In section 5 the convergence and uniqueness of the solution are proved. In section 6 the analytical example is presented to illustrate the accuracy of these methods. Finally, a brief conclusion is given in section 7 .

2. Preliminaries. The mathematical definitions of fractional derivative and fractional integration are the subject of several different approaches. The most frequently used definitions of the fractional calculus involve the Riemann-Liouville fractional derivative, Caputo derivative, Riesz derivative and Grünewald - Letnikov fractional derivative [6, 8, 16, 18, 22. In our work the Caputo's definition of fractional derivative has been used.

Definition 1. The Riemann-Liouville fractional integral of order $\alpha>0$ of a function $f$ is defined as

$$
\begin{aligned}
J^{\alpha} f(x) & =\frac{1}{\Gamma(\alpha)} \int_{0}^{x}(x-t)^{\alpha-1} f(t) d t, \quad x>0, \quad \alpha \in \mathbb{R}^{+}, \\
J^{0} f(x) & =f(x),
\end{aligned}
$$

where $\mathbb{R}^{+}$is the set of positive real numbers.

Definition 2. The fractional derivative of $f(x)$ in the Caputo sense is defined by

$$
\begin{aligned}
{ }^{c} D^{\alpha} f(x) & =J^{n-\alpha} D^{n} f(x)= \\
& = \begin{cases}\frac{1}{\Gamma(n-\alpha)} \int_{0}^{x}(x-t)^{n-\alpha-1} \frac{d^{n} f(t)}{d t^{n}} d t, & n-1<\alpha<n, \\
\frac{d^{n} f(x)}{d x^{n}}, & \alpha=n,\end{cases}
\end{aligned}
$$

where the parameter $\alpha$ is the order of the derivative and is allowed to be real or even complex. 
In this paper only real and positive $\alpha$ will be considered. Hence, we have the following properties:

- $J^{\alpha} J^{v} f=J^{\alpha+v} f, \quad \alpha, v>0$.

- $J^{\alpha} x^{\beta}=\frac{\Gamma(\beta+1)}{\Gamma(\alpha+\beta+1)} x^{\alpha+\beta}, \quad \alpha>0, \beta>-1, \quad x>0$.

- $J^{\alpha} D^{\alpha} f(x)=f(x)-\sum_{k=0}^{n-1} f^{(k)}\left(0^{+}\right) \frac{x^{k}}{k !}, \quad x>0, \quad n-1<\alpha \leq n$.

Definition 3. The Riemann-Liouville fractional derivative of order $\alpha>0$ is normally defined as

$$
D^{\alpha} f(x)=D^{n} J^{n-\alpha} f(x), \quad n-1<\alpha \leq n .
$$

Definition 4. The Laplace transform of a function $f(x)$, is defined as

$$
\mathcal{L}[f(x)]=F(s)=\int_{0}^{+\infty} f(x) e^{-s x} d x
$$

where $x>0, s$ can be either real or complex.

Definition 5. Given two functions $f$ and $g$, we define, for any $x>0$,

$$
(f * g)(x)=\int_{0}^{x} f(t) g(x-t) d t
$$

The function $f * g$ is called the convolution of $f$ and $g$.

Theorem 1. The Laplace transform of the Caputo derivative is defined by

$$
\mathcal{L}\left[{ }^{c} D^{\alpha} f(x)\right]=s^{\alpha} F(s)-\sum_{k=0}^{n-1} s^{\alpha-k-1} f^{(k)}(0), \quad n-1<\alpha \leq n .
$$

3. Adomian Decomposition Method. Consider the equation (1) where ${ }^{c} D^{\alpha}$ is the operator defined as $(3)$. Operating with $J^{\alpha}$ on both sides of the equation (1), we get $[1,2,5,7,18$.

$$
y(x)=\sum_{k=0}^{n-1} y^{(k)}\left(0^{+}\right) \frac{x^{k}}{k !}+J^{\alpha}(g(x))+
$$




$$
+J^{\alpha}\left(a(x) y(x)+\int_{0}^{x} K_{1}(x, t) F_{1}(y(t)) d t+\int_{0}^{1} K_{2}(x, t) F_{2}(y(t)) d t\right) .
$$

Adomian decomposition method defines the solution $y(x)$ by the series

$$
y=\sum_{n=0}^{\infty} y_{n}
$$

and the nonlinear functions $F_{1}$ and $F_{2}$ are decomposed as

$$
F_{1}=\sum_{n=0}^{\infty} A_{n}, \quad F_{2}=\sum_{n=0}^{\infty} B_{n}
$$

where $A_{n}, B_{n}$ are the Adomian polynomials given by

$$
\begin{aligned}
& A_{n}=\frac{1}{n !}\left[\frac{d^{n}}{d \phi^{n}} F_{1}\left(\sum_{i=0}^{n} \phi^{i} y_{i}\right)\right]_{\phi=0}, \\
& B_{n}=\frac{1}{n !}\left[\frac{d^{n}}{d \phi^{n}} F_{2}\left(\sum_{i=0}^{n} \phi^{i} y_{i}\right)\right]_{\phi=0} .
\end{aligned}
$$

The Adomian polynomials were introduced in 1 , 2, 10, 11, 13, as:

$$
\begin{aligned}
& A_{0}=F_{1}\left(y_{0}\right) \\
& A_{1}=y_{1} F_{1}^{\prime}\left(y_{0}\right) \\
& A_{2}=y_{2} F_{1}^{\prime}\left(y_{0}\right)+\frac{1}{2} y_{1}^{2} F_{1}^{\prime \prime}\left(y_{0}\right) \\
& A_{3}=y_{3} F_{1}^{\prime}\left(y_{0}\right)+y_{1} y_{2} F_{1}^{\prime \prime}\left(y_{0}\right)+\frac{1}{3} y_{1}^{3} F_{1}^{\prime \prime \prime}\left(y_{0}\right),
\end{aligned}
$$

and

$$
\begin{aligned}
B_{0} & =F_{2}\left(y_{0}\right), \\
B_{1} & =y_{1} F_{2}^{\prime}\left(y_{0}\right), \\
B_{2} & =y_{2} F_{2}^{\prime}\left(y_{0}\right)+\frac{1}{2} y_{1}^{2} F_{2}^{\prime \prime}\left(y_{0}\right),
\end{aligned}
$$




$$
B_{3}=y_{3} F_{2}^{\prime}\left(y_{0}\right)+y_{1} y_{2} F_{2}^{\prime \prime}\left(y_{0}\right)+\frac{1}{3} y_{1}^{3} F_{2}^{\prime \prime \prime}\left(y_{0}\right)
$$

The components $y_{0}, y_{1}, y_{2}, \ldots$ are determined recursively by

$$
\begin{aligned}
y_{0} & =\sum_{k=0}^{n-1} y^{(k)}\left(0^{+}\right) \frac{x^{k}}{k !}+J^{\alpha} g(x), \\
y_{k+1} & =J^{\alpha}\left(a(x) y(x)+\int_{0}^{x} K_{1}(x, t) A_{k} d t+\int_{0}^{1} K_{2}(x, t) B_{k} d t\right) .
\end{aligned}
$$

Having defined the components $y_{0}, y_{1}, y_{2}, \ldots$, the solution $y$ in a series form defined by (5) follows immediately. It is important to note that the decomposition method suggests that the $0^{t h}$ component $y_{0}$ is to be defined by the initial conditions and the function $g(x)$ as described above. The other components, namely $y_{1}, y_{2}, \ldots$, are derived recurrently.

4. Modified Laplace Adomian Decomposition Method. We consider the fractional Volterra-Fredholm integro-differential equation (1). We apply the Laplace transform to both sides [8, 9, 22]:

$$
\begin{aligned}
\mathcal{L}\left[{ }^{c} D^{\alpha} y(x)\right] & =\mathcal{L}[g(x)]+\mathcal{L}[a(x) y(x)]+ \\
& +\mathcal{L}\left[\int_{0}^{x} K_{1}(x, s) F_{1}(y(s)) d s+\int_{0}^{1} K_{2}(x, s) F_{2}(y(s)) d s\right] .
\end{aligned}
$$

Using the differentiation property of Laplace transform (4), we get

$$
\begin{aligned}
s^{\alpha} \mathcal{L}[y(x)]-y_{0} & =\mathcal{L}[g(x)]+\mathcal{L}[a(x) y(x)]+ \\
& +\mathcal{L}\left[\int_{0}^{x} K_{1}(x, s) F_{1}(y(s)) d s+\int_{0}^{1} K_{2}(x, s) F_{2}(y(s)) d s\right],
\end{aligned}
$$

where $y_{0}=\sum_{k=0}^{m-1} x^{\alpha-k-1} y^{(k)}(0)$. Thus, the given equation is equivalent to

$$
\mathcal{L}[y(x)]=\frac{y_{0}}{s^{\alpha}}+\frac{1}{s^{\alpha}} \mathcal{L}[g(x)]+\frac{1}{s^{\alpha}} \mathcal{L}[a(x) y(x)]+
$$




$$
+\frac{1}{s^{\alpha}} \mathcal{L}\left[\int_{0}^{x} K_{1}(x, s) F_{1}(y(s)) d s+\int_{0}^{1} K_{2}(x, s) F_{2}(y(s)) d s\right] .
$$

Substituting (5) and (6) into (9), we have

$$
\begin{aligned}
\mathcal{L}\left[\sum_{n=0}^{\infty} y_{n}(x)\right] & =\frac{y_{0}}{s^{\alpha}}+\frac{1}{s^{\alpha}} \mathcal{L}[g(x)]+\frac{1}{s^{\alpha}} \mathcal{L}[a(x) y(x)]+\frac{1}{s^{\alpha}} \mathcal{L} \times \\
& \times\left[\int_{0}^{x} K_{1}(x, s) \sum_{n=0}^{\infty} A_{n} d s+\int_{0}^{1} K_{2}(x, s) \sum_{n=0}^{\infty} B_{n} d s\right] .
\end{aligned}
$$

Matching both sides of 10 yields the following iterative algorithm:

$$
\begin{aligned}
\mathcal{L}\left[y_{0}(x)\right] & =\frac{y_{0}}{s^{\alpha}}+\frac{1}{s^{\alpha}} \mathcal{L}[g(x)] \\
\mathcal{L}\left[y_{1}(x)\right] & =\frac{1}{s^{\alpha}} \mathcal{L}\left[a(x) y(x)+\int_{0}^{x} K_{1}(x, s) A_{0} d s+\int_{0}^{1} K_{2}(x, s) B_{0} d s\right], \\
\mathcal{L}\left[y_{2}(x)\right] & =\frac{1}{s^{\alpha}} \mathcal{L}\left[a(x) y(x)+\int_{0}^{x} K_{1}(x, s) A_{1} d s+\int_{0}^{1} K_{2}(x, s) B_{1} d s\right], \\
& \vdots \\
\mathcal{L}\left[y_{n+1}(x)\right] & =\frac{1}{s^{\alpha}} \mathcal{L}\left[a(x) y(x)+\int_{0}^{x} K_{1}(x, s) A_{n} d s+\int_{0}^{1} K_{2}(x, s) B_{n} d s\right] .
\end{aligned}
$$

The solution $y(x)$ is defined by the series

$$
y(x)=\sum_{n=0}^{\infty} y_{n}(x)
$$

5. Uniqueness and Convergence. In this section we present an existence and uniqueness results of equation (1) with the initial condition (2) and prove it. Before starting and proving the main results, we introduce the following hypotheses: 
(A1) There exist two constants $L_{F_{1}}, L_{F_{2}}>0$ such that, for any $y_{1}$ and $y_{2} \in C(J, \mathbb{R})$

$$
\left|F_{1}\left(y_{1}(x)\right)-F_{1}\left(y_{2}(x)\right)\right| \leq L_{F_{1}}\left|y_{1}-y_{2}\right|
$$

and

$$
\left|F_{2}\left(y_{1}(x)\right)-F_{2}\left(y_{2}(x)\right)\right| \leq L_{F_{2}}\left|y_{1}-y_{2}\right| .
$$

(A2) There exist two functions $K_{1}^{*}, K_{2}^{*} \in C\left(D, \mathbb{R}^{+}\right)$, where $C\left(D, \mathbb{R}^{+}\right)$is the set of all positive functions, continuous on $D=\{(x, t) \in \mathbb{R} \times \mathbb{R}$ : $0 \leq t \leq x \leq 1\}$, such that

$$
K_{1}^{*}=\sup _{x \in[0,1]} \int_{0}^{x}\left|K_{1}(x, t)\right| d t<\infty, \quad K_{2}^{*}=\sup _{x \in[0,1]} \int_{0}^{1}\left|K_{2}(x, t)\right| d t<\infty .
$$

(A3) The two functions $a, g: J \rightarrow \mathbb{R}$ are continuous.

Lemma 1. If $y_{0} \in C(J, \mathbb{R})$, then $y(x) \in C\left(J, \mathbb{R}^{+}\right)$is a solution of the problem (1) - 2) iff $y$ satisfies

$$
\begin{aligned}
y(x) & =\frac{1}{\Gamma(\alpha)} \int_{0}^{x}(x-s)^{\alpha-1} a(s) y(s) d s+\frac{1}{\Gamma(\alpha)} \int_{0}^{x}(x-s)^{\alpha-1} g(s) d s+ \\
& +\frac{1}{\Gamma(\alpha)} \int_{0}^{x}(x-s)^{\alpha-1}\left[\int_{0}^{s} K_{1}(s, \tau) F_{1}(y(\tau)) d \tau+\right. \\
& \left.+\int_{0}^{1} K_{2}(s, \tau) F_{2}(y(\tau)) d \tau\right] d s+y_{0}
\end{aligned}
$$

for $x \in J$.

Our first result is based on the Banach contraction principle.

Theorem 2. Assume that (A1), (A2) and (A3) hold. If

$$
\left(\frac{\|a\|_{\infty}+K_{1}^{*} L_{F_{1}}+K_{2}^{*} L_{F_{2}}}{\Gamma(\alpha+1)}\right)<1
$$

then there exists a unique solution $y(x) \in C(J)$ of (1) - (2). 
Proof. By Lemma 1 we know that $y$ is a solution of $(1)-(2)$ iff it satisfies

$$
\begin{aligned}
y(x) & =\frac{1}{\Gamma(\alpha)} \int_{0}^{x}(x-s)^{\alpha-1} a(s) y(s) d s+\frac{1}{\Gamma(\alpha)} \int_{0}^{x}(x-s)^{\alpha-1} g(s) d s+ \\
& +\frac{1}{\Gamma(\alpha)} \int_{0}^{x}(x-s)^{\alpha-1}\left[\int_{0}^{s} K_{1}(s, \tau) F_{1}(y(\tau)) d \tau+\right. \\
& \left.+\int_{0}^{1} K_{2}(s, \tau) F_{2}(y(\tau)) d \tau\right] d s+y_{0} .
\end{aligned}
$$

Let the operator $T: C(J, \mathbb{R}) \rightarrow C(J, \mathbb{R})$ be defined by

$$
\begin{aligned}
(T y)(x) & =\frac{1}{\Gamma(\alpha)} \int_{0}^{x}(x-s)^{\alpha-1} a(s) y(s) d s+\frac{1}{\Gamma(\alpha)} \int_{0}^{x}(x-s)^{\alpha-1} g(s) d s+ \\
& +\frac{1}{\Gamma(\alpha)} \int_{0}^{x}(x-s)^{\alpha-1}\left[\int_{0}^{s} K_{1}(s, \tau) F_{1}(y(\tau)) d \tau+\right. \\
& \left.+\int_{0}^{1} K_{2}(s, \tau) F_{2}(y(\tau)) d \tau\right] d s+y_{0} .
\end{aligned}
$$

We can see that, if $y \in C(J, \mathbb{R})$ is a fixed point of $T$, then $y$ is a solution of $(1)-2$.

Now we prove that $T$ has a fixed point $y$ in $C(J, \mathbb{R})$. For that, let $y_{1}, y_{2} \in C(J, \mathbb{R})$ be such that for any $x \in[0,1]$

$$
\begin{aligned}
y_{1}(x) & =\frac{1}{\Gamma(\alpha)} \int_{0}^{x}(x-s)^{\alpha-1} a(s) y_{1}(s) d s+\frac{1}{\Gamma(\alpha)} \int_{0}^{x}(x-s)^{\alpha-1} g(s) d s+ \\
& +\frac{1}{\Gamma(\alpha)} \int_{0}^{x}(x-s)^{\alpha-1}\left[\int_{0}^{s} K_{1}(s, \tau) F_{1}\left(y_{1}(\tau)\right) d \tau+\right. \\
& \left.+\int_{0}^{1} K_{2}(s, \tau) F_{2}\left(y_{1}(\tau)\right) d \tau\right] d s+y_{0},
\end{aligned}
$$




$$
\begin{aligned}
y_{2}(x) & =\frac{1}{\Gamma(\alpha)} \int_{0}^{x}(x-s)^{\alpha-1} a(s) y_{2}(s) d s+\frac{1}{\Gamma(\alpha)} \int_{0}^{x}(x-s)^{\alpha-1} g(s) d s+ \\
& +\frac{1}{\Gamma(\alpha)} \int_{0}^{x}(x-s)^{\alpha-1}\left[\int_{0}^{s} K_{1}(s, \tau) F_{1}\left(y_{2}(\tau)\right) d \tau+\right. \\
& \left.+\int_{0}^{1} K_{2}(s, \tau) F_{2}\left(y_{2}(\tau)\right) d \tau\right] d s+y_{0} .
\end{aligned}
$$

Consequently, we get

$$
\begin{aligned}
& \left|\left(T y_{1}\right)(x)-\left(T y_{2}\right)(x)\right| \leq \\
\leq & \frac{1}{\Gamma(\alpha)} \int_{0}^{x}(x-s)^{\alpha-1}|a(s)|\left|y_{1}(s)-y_{2}(s)\right| d s+\frac{1}{\Gamma(\alpha)} \times \\
& \times \int_{0}^{x}(x-s)^{\alpha-1}\left(\begin{array}{c}
\int_{0}^{s}\left|K_{1}(s, \tau)\right|\left|F_{1}\left(y_{1}(\tau)\right)-F_{1}\left(y_{2}(\tau)\right)\right| d \tau+ \\
+\int_{0}^{1}\left|K_{2}(s, \tau)\right|\left|F_{2}\left(y_{1}(\tau)\right)-F_{2}\left(y_{2}(\tau)\right)\right| d \tau
\end{array}\right) d s \leq \\
\leq & \frac{\|a\|_{\infty}}{\Gamma(\alpha+1)}\left|y_{1}(x)-y_{2}(x)\right|+\frac{K_{1}^{*} L_{F_{1}}}{\Gamma(\alpha+1)}\left|y_{1}(x)-y_{2}(x)\right|+ \\
& +\frac{K_{2}^{*} L_{F_{2}}}{\Gamma(\alpha+1)}\left|y_{1}(x)-y_{2}(x)\right|= \\
= & \left(\frac{\|a\|_{\infty}+K_{1}^{*} L_{F_{1}}+K_{2}^{*} L_{F_{2}}}{\Gamma(\alpha+1)}\right)\left|y_{1}(x)-y_{2}(x)\right| .
\end{aligned}
$$

From the inequality 12 we have

$$
\left\|T y_{1}-T y_{2}\right\|_{\infty} \leq\left\|y_{1}-y_{2}\right\|_{\infty} .
$$

So, $T$ is a contraction map. By the Banach contraction principle, we conclude that $T$ has an unique fixed point $y$ in $C(J, \mathbb{R})$.

Theorem 3. Suppose that (A1)-(A3) and 12 hold. If the series solution $y(x)=\sum_{i=0}^{\infty} y_{i}(x)$ and $\left\|y_{1}\right\|_{\infty}<\infty$, obtained by the $m$-order deformation, is convergent, then it converges to the exact solution of the fractional Volterra-Fredholm integro-differential equation (1) - (2). 
Proof. Denote as $\left(C[0,1],\|\cdot\|_{\infty}\right)$ the Banach space of all continuous functions on $J$, with $\left|y_{1}(x)\right| \leq \infty$ for all $x$ in $J$.

Frist we define the sequence of partial sums $s_{n}$. Let $s_{n}$ and $s_{m}$ be arbitrary partial sums with $n \geq m$. We are to prove that $s_{n}=\sum_{i=0}^{n} y_{i}(x)$ is a Cauchy sequence in this Banach space:

$$
\begin{aligned}
& \left\|s_{n}-s_{m}\right\|_{\infty}=\max _{\forall x \in J}\left|s_{n}-s_{m}\right|=\max _{\forall x \in J}\left|\sum_{i=0}^{n} y_{i}(x)-\sum_{i=0}^{m} y_{i}(x)\right|= \\
& =\max _{\forall x \in J} \mid \sum_{i=m+1}^{n}\left(\frac { 1 } { \Gamma ( \alpha ) } \int _ { 0 } ^ { x } ( x - t ) ^ { \alpha - 1 } \left[a(t) y_{i}(t)+\right.\right. \\
& \left.\left.+\int_{0}^{t} K_{1}(t, s) A_{i}(s) d s+\int_{0}^{1} K_{2}(t, s) B_{i}(s) d s\right] d t\right) \mid= \\
& \quad=\max _{\forall x \in J} \mid \frac{1}{\Gamma(\alpha)} \int_{0}^{x}(x-t)^{\alpha-1}\left[a(t) \sum_{i=m}^{n-1} y_{i}(t)+\right. \\
& \left.+\int_{0}^{t} K_{1}(t, s) \sum_{i=m}^{n-1} A_{i}(s) d s+\int_{0}^{1} K_{2}(t, s) \sum_{i=m}^{n-1} B_{i}(s) d s\right] d t \mid .
\end{aligned}
$$

From (5) and (6), we have

$$
\begin{gathered}
\sum_{i=m}^{n-1} A_{i}=F_{1}\left(s_{n-1}\right)-F_{1}\left(s_{m-1}\right), \\
\sum_{i=m}^{n-1} B_{i}=F_{2}\left(s_{n-1}\right)-F_{2}\left(s_{m-1}\right), \\
\sum_{i=m}^{n-1} y_{i}=y\left(s_{n-1}\right)-y\left(s_{m-1}\right) .
\end{gathered}
$$


So,

$$
\begin{aligned}
& \left\|s_{n}-s_{m}\right\|_{\infty}=\max _{\forall x \in J} \mid \frac{1}{\Gamma(\alpha)} \int_{0}^{x}(x-t)^{\alpha-1}\left[a(t)\left(y\left(s_{n-1}\right)-y\left(s_{m-1}\right)\right)+\right. \\
& +\int_{0}^{t} K_{1}(t, s)\left(F_{1}\left(s_{n-1}\right)-F_{1}\left(s_{m-1}\right)\right) d s+ \\
& \left.+\int_{0}^{1} K_{2}(t, s)\left(F_{2}\left(s_{n-1}\right)-F_{2}\left(s_{m-1}\right)\right) d s\right] d t \mid \leq \\
& \leq \max _{\forall x \in J}\left(\frac { 1 } { \Gamma ( \alpha ) } \int _ { 0 } ^ { x } | x - t | ^ { \alpha - 1 } \left[|a(t)|\left|y\left(s_{n-1}\right)-y\left(s_{m-1}\right)\right|+\right.\right. \\
& +\int_{0}^{t}\left|K_{1}(t, s)\right|\left|\left(F_{1}\left(s_{n-1}\right)-F_{1}\left(s_{m-1}\right)\right)\right| d s+ \\
& \left.\left.+\int_{0}^{1}\left|K_{2}(t, s)\right|\left|\left(F_{2}\left(s_{n-1}\right)-F_{2}\left(s_{m-1}\right)\right)\right| d s\right] d t\right) \leq \\
& \leq \frac{1}{\Gamma(\alpha+1)}\left[\|a(t)\|_{\infty}\left\|s_{n-1}-s_{m-1}\right\|_{\infty}+K_{1}^{*} L_{F_{1}} \times\right. \\
& \left.\times \quad\left\|s_{n-1}-s_{m-1}\right\|_{\infty}+K_{2}^{*} L_{F_{2}}\left\|s_{n-1}-s_{m-1}\right\|_{\infty}\right]= \\
& =\left(\frac{\|a\|_{\infty}+K_{1}^{*} L_{F_{1}}+K_{2}^{*} L_{F_{2}}}{\Gamma(\alpha+1)}\right)\left\|s_{n-1}-s_{m-1}\right\|_{\infty}= \\
& =\delta\left\|s_{n-1}-s_{m-1}\right\|_{\infty} \text {, }
\end{aligned}
$$

where

$$
\delta=\left(\frac{\|a\|_{\infty}+K_{1}^{*} L_{F_{1}}+K_{2}^{*} L_{F_{2}}}{\Gamma(\alpha+1)}\right) .
$$

Let $n=m+1$, then

$$
\left\|s_{n}-s_{m}\right\|_{\infty} \leq \delta\left\|s_{m}-s_{m-1}\right\|_{\infty} \leq \ldots \leq \delta^{m}\left\|s_{1}-s_{0}\right\|_{\infty}
$$


so,

$$
\begin{aligned}
\left\|s_{n}-s_{m}\right\|_{\infty} & \leq \sum_{k=m+1}^{n}\left\|s_{k}-s_{k-1}\right\|_{\infty} \leq \\
& \leq\left(\delta^{m}+\delta^{m+1}+\cdots+\delta^{n-1}\right)\left\|s_{1}-s_{0}\right\|_{\infty} \leq \\
& \leq \delta^{m}\left(1+\delta+\delta^{2}+\cdots+\delta^{n-m-1}\right)\left\|s_{1}-s_{0}\right\|_{\infty} \leq \\
& \leq \delta^{m}\left(\frac{1-\delta^{n-m}}{1-\delta}\right)\left\|y_{1}\right\|_{\infty} .
\end{aligned}
$$

Since $0<\delta<1$, we have $\left(1-\delta^{n-m}\right)<1$, then

$$
\left\|s_{n}-s_{m}\right\|_{\infty} \leq \frac{\delta^{m}}{1-\delta}\left\|y_{1}\right\|_{\infty} .
$$

As $\left|y_{1}(x)\right|<\infty$, then $\left\|s_{n}-s_{m}\right\|_{\infty} \longrightarrow 0$ when $m \longrightarrow \infty$.

We conclude that $s_{n}$ is a Cauchy sequence in $C[0,1]$, so $y=\lim _{n \rightarrow \infty} y_{n}$. Then, the series converges and the proof is completed.

6. Illustrative Example. In this section, we present the analytical techniques based on the Adomian decomposition method and the modified Laplace Adomian decomposition method to solve Caputo fractional Volterra - Fredholm integro-differential equation.

Example. Consider the following Caputo fractional Volterra-Fredholm integro-differential equation.

${ }^{c} D^{0.75}[y(t)]=\frac{6 t^{2.25}}{\Gamma(3.25)}-\frac{t^{2} e^{t}}{5} y(t)+\int_{0}^{t} e^{t} s y(s) d s+\int_{0}^{1}\left(4-s^{-3}\right) y(s) d s$,

with the initial condition $y(0)=0$ and the exact solution $y(t)=t^{3}$.

Firstly, we apply the Adomian decomposition method.

Applying the operator $J^{0.75}$ to both sides of 13 ,

$$
\begin{aligned}
y(t) & =\sum_{k=0}^{m-1} \frac{d y(0)}{d t^{k}} \frac{t^{k}}{k !}+\frac{6}{\Gamma(3.25)} J^{0.75}\left[t^{2.25}\right]-\frac{1}{5} J^{0.75}\left[t^{2} e^{t} y(t)\right]+ \\
& +J^{0.75}\left[\int_{0}^{t} e^{t} s y(s) d s+\int_{0}^{1}\left(4-s^{-3}\right) y(s) d s\right] .
\end{aligned}
$$


Then,

$$
\begin{aligned}
& y_{0}(t)=\sum_{k=0}^{m-1} \frac{d y(0)}{d t^{k}} \frac{t^{k}}{k !}+\frac{6}{\Gamma(3.25)} J^{0.75} t^{2.25}= \\
& =0+\frac{6}{\Gamma(3.25)} \frac{\Gamma(9 / 4+1)}{\Gamma(9 / 4+3 / 4+1)} t^{(9 / 4+3 / 4)}=t^{3}, \\
& y_{1}(t)=J^{0.75}\left[\frac{-t^{2} e^{t} y_{0}(t)}{5}+\int_{0}^{t} e^{t} s y_{0}(s) d s+\int_{0}^{1}\left(4-s^{-3}\right) y_{0}(s) d s\right]= \\
& =-\frac{1}{5} J^{0.75}\left[t^{2} e^{t} y_{0}(t)\right]+J^{0.75}\left[\int_{0}^{t} e^{t} s^{4} d s+\int_{0}^{1}\left(4-s^{-3}\right) s^{3} d s\right]= \\
& =-\frac{1}{5} J^{0.75}\left[t^{2} e^{t} y_{0}(t)\right]+J^{0.75}\left[\frac{1}{5} e^{t} t^{5}+0\right]= \\
& =-\frac{1}{5} J^{0.75}\left[t^{2} e^{t} y_{0}(t)\right]+\frac{1}{5} J^{0.75}\left[e^{t} t^{2} y_{0}(t)\right]=0 \text {, } \\
& y_{n}(t)=0 \text {. }
\end{aligned}
$$

Therefore, the obtained solution is

$$
y(t)=t^{3} .
$$

Secondly, the modified Laplace Adomian decomposition method.

We apply the Laplace transform to both sides of 13 .

$$
\begin{aligned}
\mathcal{L}\left[{ }^{c} D^{0.75} y(t)\right] & =\mathcal{L}\left[\left(-\frac{t^{2} e^{t}}{5}\right) y(t)\right]+\mathcal{L}\left[\frac{6 t^{2.25}}{\Gamma(3.25)}\right]+ \\
& +\mathcal{L}\left[\int_{0}^{t} e^{t} s y(s) d s+\int_{0}^{1}\left(4-s^{-3}\right) y(s) d s\right] .
\end{aligned}
$$

Using the property of Laplace transform and the initial condition, we get

$$
s^{\frac{3}{4}} \mathcal{L}[y(t)]=\mathcal{L}\left[\left(-\frac{t^{2} e^{t}}{5}\right) y(t)\right]+\mathcal{L}\left[\frac{6 t^{2.25}}{\Gamma(3.25)}\right]+
$$




$$
\begin{aligned}
& +\mathcal{L}\left[\int_{0}^{t} e^{t} s y(s) d s+\int_{0}^{1}\left(4-s^{-3}\right) y(s) d s\right], \\
\mathcal{L}[y(t)]= & \frac{1}{s^{\frac{3}{4}}} \mathcal{L}\left[\left(-\frac{t^{2} e^{t}}{5}\right) y(t)\right]+\frac{1}{s^{\frac{3}{4}}} \mathcal{L}\left[\frac{6 t^{2.25}}{\Gamma(3.25)}\right] \\
+ & \frac{1}{s^{\frac{3}{4}}} \mathcal{L}\left[\int_{0}^{t} e^{t} s y(s) d s+\int_{0}^{1}\left(4-s^{-3}\right) y(s) d s\right] .
\end{aligned}
$$

Substituting (5) and (6) into the above equation, we obtain

$$
\begin{aligned}
\mathcal{L}\left[\sum_{n=0}^{\infty} y_{n}(t)\right] & =\frac{1}{s^{\frac{3}{4}}} \mathcal{L}\left[\frac{6 t^{2.25}}{\Gamma(3.25)}\right]+\frac{1}{s^{\frac{3}{4}}} \mathcal{L}\left[\left(-\frac{t^{2} e^{t}}{5}\right) \sum_{n=0}^{\infty} y_{n}(t)\right]+ \\
& +\frac{1}{s^{\frac{3}{4}}} \mathcal{L}\left[\int_{0}^{t} e^{t} s \sum_{n=0}^{\infty} A_{n} d s+\int_{0}^{1}\left(4-s^{-3}\right) \sum_{n=0}^{\infty} B_{n} d s\right] .
\end{aligned}
$$
tion:

By matching both sides of above equation, we have the following rela-

$$
\begin{aligned}
& \mathcal{L}\left[y_{0}(t)\right]=\frac{1}{s^{\frac{3}{4}}} \mathcal{L}\left[\frac{6 t^{2.25}}{\Gamma(3.25)}\right], \\
& \mathcal{L}\left[y_{1}(t)\right]=\frac{1}{s^{\frac{3}{4}}} \mathcal{L}\left[\frac{-t^{2} e^{t} y_{0}(t)}{5}+\int_{0}^{t} e^{t} s A_{0} d s+\int_{0}^{1}\left(4-s^{-3}\right) B_{0} d s\right],
\end{aligned}
$$$$
\mathcal{L}\left[y_{n+1}(t)\right]=\frac{1}{s^{\frac{3}{4}}} \mathcal{L}\left[\frac{-t^{2} e^{t} y_{n}(t)}{5}+\int_{0}^{t} e^{t} s A_{n} d s+\int_{0}^{1}\left(4-s^{-3}\right) B_{n} d s\right] .
$$

Applying inverse Laplace transform to above equations, we get

$$
\begin{aligned}
& y_{0}(t)=t^{3} \\
& y_{1}(t)=\mathcal{L}^{-1}\left[\frac{1}{s^{\frac{3}{4}}} \mathcal{L}\left[\frac{-t^{2} e^{t} y_{0}(t)}{5}+\int_{0}^{t} e^{t} s^{4} d s+\int_{0}^{1}\left(4-s^{-3}\right) s^{3} d s\right]\right]=0
\end{aligned}
$$




$$
y_{n}(t)=0 \text {. }
$$

Therefore, the obtained solution is $y(t)=t^{3}$.

7. Conclusion. In this paper, Adomian decomposition method and modified Laplace Adomian decomposition method have been successfully applied to find the approximate solution of the fractional VolterraFredholm integro-differential equation. The reliability of the methods and reduction in the size of the computational work give these methods a wider applicability. The methods are very powerful and efficient in finding analytical as well as numerical solutions for wide classes of linear and nonlinear fractional Volterra-Fredholm integro-differential equations. They provide more realistic series solutions that converge very rapidly in real physical problems. Finally, the behavior of the solution can be formally determined by analytical approximate. The proposed methods can be applied to other nonlinear fractional differential equations, systems of differential and integral equations.

\section{References}

[1] Abbaoui K., Cherruault Y. Convergence of Adomian's method applied to nonlinear equations. Math. Comput. Model., 1994, vol. 20, no. 9, pp. 6973. DOI: 10.1016/0895-7177(94)00163-4.

[2] Adomian G. A review of the decomposition method in applied mathematics. J. Math. Anal. Appl., 1988, vol. 135, no. 2, pp. 501-544. DOI: 10.1016/0022-247X(88)90170-9.

[3] AL-Smadi M., Gumah G. On the homotopy analysis method for fractional SEIR epidemic model. Res. J. Appl. Sci. Eng. Technol., 2014, vol. 18, no. 7 , pp. 3809-3820.

[4] Alhendi F., Shammakh W., Al-Badrani H. Numerical solutions for quadratic integro-differential equations of fractional orders. Open J. Appl. Sci., 2017, vol. 7, pp. 157-170. DOI: 10.4236/ojapps.2017.74014.

[5] Araghi M., Behzadi S. Solving nonlinear Volterra-Fredholm integrodifferential equations using the modified Adomian decomposition method. Comput. Methods Appl. Math., 2009, vol. 9, no. 4, pp. 321-331. DOI: 10.2478/cmam-2009-0020.

[6] Daftardar-Gejji V., Jafari H. Solving a multi-order fractional differential equation using Adomian decomposition. Appl. Math. Comput., 2007, vol. 189, no. 1, pp. 541-548. DOI: 10.1016/j.amc.2006.11.129. 
[7] Ghadle K., Hamoud A. Study of the approximate solution of fuzzy Volterra - Fredholm integral equations by using (ADM), Elixir Appl. Math., 2016, vol. 98, pp. 42567-42573.

[8] Hamoud A., Ghadle K. The reliable modified of Laplace Adomian decomposition method to solve nonlinear interval Volterra-Fredholm integral equations. Korean J. Math., 2017, vol. 25, no. 3, pp. 323-334. DOI: 10.11568/kjm.2017.25.3.323.

[9] Hamoud A., Ghadle K. The combined modified Laplace with Adomian decomposition method for solving the nonlinear Volterra-Fredholm integrodifferential equations. J. Korean Soc. Ind. Appl. Math., 2017, vol. 21, no. 1, pp. 17-28. DOI: 10.12941/jksiam.2017.21.017.

[10] Hamoud A., Ghadle K. Modified Adomian decomposition method for solving fuzzy Volterra-Fredholm integral equations. J. Indian Math. Soc., 2018, vol. 85, no. (1-2), pp. 01-17. DOI: 10.18311/jims/2018/16260.

[11] Hamoud A., Ghadle K. A study of some reliable methods for solving fuzzy Volterra-Fredholm integral equations. Acta Universitatis Apulensis, 2018, vol. 53, pp. 65-92. DOI: 10.17114/j.aua.2018.53.06.

[12] Hamoud A., Ghadle K. Recent advances on reliable methods for solving Volterra-Fredholm integral and integro-differential equations. Asian Journal of Mathematics and Computer Research, 2018, vol. 24, pp. 128-157.

[13] Hamoud A., Ghadle K. Existence and uniqueness results for fractional Volterra-Fredholm integro-differential equations. Int. J. Open Problems Compt. Math., 2018, vol. 11, no. 3, pp. 16-30. To Appear.

[14] Jafarian A., Rostami F., Golmankhaneh A., Baleanu D. Using ANNs approach for solving fractional order Volterra integro-differential equations. Int. J. Comput. Intell. Syst., 2017, vol. 10, pp. 470-480. DOI: 10.2991/ijcis.2017.10.1.32.

[15] Kadem A., Kilicman A. The approximate solution of fractional Fredholm integro-differential equations by variational iteration and homotopy perturbation methods. Abstr. Appl. Anal., 2012, vol. 2012, pp. 1-10. DOI: 10.1155/2012/486193.

[16] Kilbas A., Srivastava H., Trujillo J. Theory and applications of fractional differential equations. North-Holland Math. Stud., 2006.

[17] Ma X., Huang C. Numerical solution of fractional integro-differential equations by a hybrid collocation method. Appl. Math. Comput., 2013, vol. 219, no. 12, pp. 6750-6760. DOI: 10.1016/j.amc.2012.12.072.

[18] Mittal R., Nigam R. Solution of fractional integro-differential equations by Adomian decomposition method. Int. J. Appl. Math. Mech., 2008, vol. 4, no. 2, pp. $87-94$. 
[19] Momani S., Noor M. Numerical methods for four-order fractional integrodifferential equations. Appl. Math. Comput., 2006, vol. 182, pp. 754-760. DOI: $10.1016 /$ j.amc.2006.04.041.

[20] Momani S., Qaralleh R. An efficient method for solving systems of fractional integro-differential equations. Comput. Math. Appl., 2006, vol. 52, pp. 459-470. DOI: 10.1016/j.camwa.2006.02.011.

[21] Yang C. Numerical solution of nonlinear Fredholm integro-differential equations of fractional order by using hybrid of block-pulse functions and Chebyshev polynomials. Math. Probl. Eng., 2011, vol. 2011, pp. 1-11. DOI: $10.1155 / 2011 / 341989$.

[22] Yang C., Hou J. Numerical solution of integro-differential equations of fractional order by Laplace decomposition method. Wseas Trans. Math., 2013, vol. 12, no. 12, pp. 1173-1183.

Received January 19, 2018.

In revised form, May 12, 2018.

Accepted May 14, 2018.

Published online May 25, 2018.

Ahmed A. Hamoud

Department of Mathematics,

Dr. Babasaheb Ambedkar Marathwada University,

Aurangabad, 431004, India;

Department of Mathematics,

Taiz University, Taiz, Yemen.

E-mail: drahmedselwi985@gmail.com

Kirtiwant P. Ghadle

Department of Mathematics,

Dr. Babasaheb Ambedkar Marathwada University,

Aurangabad, 431004, India.

E-mail: drkp.ghadle@gmail.com 